\title{
A NOTE ON $M$-IDEALS IN $B(X)$
}

\author{
JULIEN HENNEFELD
}

\begin{abstract}
In this paper we prove some properties of $M$-ideals and HB-subspaces in an arbitrary Banach space. We then apply these properties to prove a theorem which generalizes to other spaces Smith's and Ward's results in [8]: for $1<p<\infty, B\left(l_{p}\right)$ contains no nontrivial summands and that each nontrivial $M$-ideal in $B\left(l_{p}\right)$ contains $K\left(l_{p}\right)$.
\end{abstract}

Introduction. A closed subspace $J$ of a Banach space $Y$ is said to be an $M$-ideal of $Y$ if its annihilator $J^{\perp}$ is $l_{1}$ complemented in $Y^{*}$. That is, there exists a subspace $J_{*}$ of $Y^{*}$ such that $Y^{*}=J^{\perp} \oplus J_{*}$ and $\|p+q\|=\|p\|+$ $\|q\|$ whenever $p \in J^{\perp}$ and $q \in J_{*} . J$ is said to be an $M$-summand if $J$ is complemented by a closed subspace $J^{\prime}$ such that $\|p+q\|=\max (\|p\|,\|q\|)$ whenever $p \in J$ and $q \in J^{\prime} . M$-summands are $M$-ideals, though the reverse is not necessarily true. These concepts, first introduced for real Banach spaces in [1], also apply to complex Banach spaces. Recently, much interest has focused on the approximation properties of $M$-ideals [5], [7].

For a Banach space $X$, let $K(X)$ and $B(X)$ denote the spaces of compact operators and all bounded operators respectively. In [3], Hennefeld showed that for $X=c_{0}$ or $l_{p}, 1<p<\infty, K(X)$ is an $M$-ideal in $B(X)$. In [8], Smith and Ward proved that, for $1<p<\infty, B(X)$ contains no nontrivial $M$ summands, and that any nontrivial $M$-ideal must contain $K\left(l_{p}\right)$. Their proof used Tam's characterization of Hermitian operators in $B\left(l_{p}\right), p \neq 2$, the fact that $K\left(l_{p}\right)$ is the only two-sided ideal in $B\left(l_{p}\right)$, and their technique of investigating Banach algebra (with identity) $M$-ideals by looking at the associated Hermitian projections (this technique involves consideration of $B\left(l_{p}\right)^{* *}$ and the Arens multiplication). In our proof of the generalization of the Smith-Ward result, we use instead some elementary properties of $M$ ideals and HB-subspaces, given in $\$ 1$, and certain manipulations on matrices.

1. Some properties of $M$-ideals and HB-subspaces. The notion of HB-subspaces, first defined in [4], is a generalization of $M$-ideals. Moreover, in [4], it was shown that for certain Banach spaces $K(X)$ is only an HB-subspace, not an $M$-ideal, in $B(X)$.

Definition 1.1. A closed subspace $H$ of a Banach space $Y$ is called an HB-subspace if its annihilator $H^{\perp}$ is complemented by a subspace $H_{*}$ such that for each $f \in Y^{*},\|f\| \geqslant\left\|f_{\perp}\right\|$ and $\|f\|>\left\|f_{*}\right\|$ whenever $f=f_{*}+f_{\perp}$ with $f_{*} \in H_{*}$ and $f_{\perp}$ nonzero $\in H^{\perp}$.

Received by the editors February 19, 1978 and, in revised form, January 3, 1979.

AMS (MOS) subject classifications (1970). Primary 46B99, 46C05, 47B05.

Key words and phrases. $M$-ideals, basis, compact operators. 
We then have the following straightforward lemmas, some of which we merely state without proof.

LEMMA 1.2. If $H$ is an $H B$-subspace of $Y$, then each $\phi \in H^{*}$ has a unique norm-preserving extension to $Y$.

LeMMA 1.3. Let $H$ be an $H B$-subspace. Then $f \in H_{*} \Leftrightarrow\|f / H\|=\|f\|$.

Proof. $(\Leftarrow)$ Let $f$ satisfy $\|f / H\|=\|f\|$. Write $f=f_{*}+f_{\perp}$. For $\varepsilon>0, \exists$ norm one $x \in H:\|f\|-\varepsilon<f(x)=f_{*}(x)+f_{\perp}(x)=f_{*}(x)$. Hence, $\left\|f_{*}\right\|=$ $\|f\|,\left\|f_{\perp}\right\|=0$ and $f=f_{*}$.

$(\Rightarrow)$ For $f \in H_{*}$, let $g=f / H$ and $\hat{g}$ be a Hahn-Banach extension of $g$ to $Y$. By the previous part of the proof, $\hat{g} \in H_{*}$. But $f-g$ is in both $H_{*}$ and $H^{\perp}$, which implies $f-\hat{g}=0$. Thus, $\|f / H\|=\|f\|$.

The proof of the above lemma shows how to obtain the decomposition for an arbitrary $g \in Y^{*}$, namely: $g_{*}$ is the unique Hahn-Banach extension of $g$ restricted to $H$, and $g_{\perp}=g-g_{*}$. Hence, we have the following lemma.

LeMMA 1.4. If $H$ is an $H B$-subspace, then $H_{*}$ is isometric to $H^{*}$.

LEMMA 1.5. If $H$ is an $H B$-subspace, and $J$ is an $M$-ideal with $H_{*} \subset J_{*}$, then $H \subset J$.

Proof. First, we claim that $J^{\perp} \subset H^{\perp}$. To see this, suppose $g \neq 0$ is in $J^{\perp}$. Write $g=g_{H_{*}}+g_{H_{\perp}}$. Note that $g_{H_{\perp}}$ cannot be 0 , since $H_{*} \subset J_{*}$; also if $g_{H_{*}}=0$, then we are finished. Hence, we can suppose $g_{H_{*}}$ and $g_{H_{\perp}}$ are both nonzero. Then,

$$
\begin{aligned}
\left\|-g_{H_{*}}+g\right\| & =\left\|g_{H_{\perp}}\right\|<\left\|-g_{H_{*}}\right\|+\left\|g_{H_{\perp}}\right\| \quad \text { (since } g_{H_{*}} \text { is nonzero) } \\
& \leqslant\left\|-g_{H_{*}}\right\|+\|g\| \quad \text { (since } H \text { is an HB-subspace). }
\end{aligned}
$$

But $-g_{H} \in J_{*}, g \in J^{\perp}$ contradicts the fact that $J$ is an $M$-ideal. Hence, $J^{\perp \perp} \subset \ddot{H}^{\perp \perp}$. Finally, $\boldsymbol{H} \subset J$, since $\boldsymbol{H}=H^{\perp \perp} \cap Y, J=J^{\perp \perp} \cap Y$.

LEMMA 1.6. Let $J$ be an $M$-ideal of $Y$ and $f \in Y^{*}$. Then $f$ is an extreme point of the unit ball of $Y^{*} \Leftrightarrow f$ is in $J_{*}$ or $J^{\perp}$ and is an extreme point of the unit ball of $J_{*}$ or $J^{\perp}$.

LEMMA 1.7. Let $H$ be an $H B$-subspace and $J$ an $M$-ideal. If $f \in H_{*}$ is an extreme point of the unit ball of $H_{*}$, then $f$ is in $J_{*}$ or $J^{\perp}$.

\section{The generalization.}

Definition 2.1. A basis $\left\{e_{i}\right\}$ is called shrinking if the biorthogonal functionals $\left\{e_{i}^{*}\right\}$ form a basis for $X^{*}$.

Definition 2.2. A basis $\left\{e_{i}\right\}$ for a Banach space is called unconditionally monotone if $\left\|\sum_{i \in A \cup B} a_{i} e_{i}\right\| \geqslant\left\|\sum_{i \in A} a_{i} e_{i}\right\|$ for all $A$ and $B$.

If $X$ has a shrinking basis $\left\{e_{i}\right\}$, then it follows from [6] that the operators with finite matrices are norm dense in $K(X)$. Hence, in this case, we can associate a matrix to each $f \in K(X)^{*}$ such that $f$ is determined by its matrix. 
LEMMA 2.3. Let $X$ have an unconditionally monotone, shrinking basis.

(1) For each $f \in K(X)^{*}$, the functional obtained from the matrix of $f$ by replacing with zeros any set of rows or columns will have norm $<\|f\|$.

(2) If a matrix in $K(X)$ consists of a single nonzero column (row), its norm in $K(X)$ is equal to its norm as an element of $X\left(X^{*}\right)$.

(3) If a matrix in $K(X)^{*}$ consists of a single nonzero column (row), its norm in $K(X)^{*}$ is equal to its norm as an element of $X^{*}\left(X^{* *}\right)$.

These facts are proved in [2].

Definition 2.4. We shall call a basis $\left\{e_{i}\right\}$ uniformly smooth if, for each $\varepsilon>0, \exists \delta>0$ such that $\|x+y\|<1+\varepsilon\|y\|$ whenever $x$ and $y$ have disjoint supports, $\|x\|=1$ and $\|y\|<\delta$. We shall call $\left\{e_{i}\right\}$ quasi-uniformly smooth if, for each $\varepsilon>0, \exists \delta>0$ such that $\left\|e_{i}+\lambda e_{j}\right\|<1+\delta \varepsilon$ for all $i, j$, whenever $|\lambda|<\delta$. Note that if a basis is uniformly smooth, the Banach space itself need not be uniformly smooth. For example, consider the standard basis for $c_{0}$.

The following is a generalization of the Smith-Ward result, since the hypotheses of the theorem are satisfied if $X$ is $l_{p}, 1<p<\infty$.

THEOREM 2.5. Let $X$ be a Banach space with an unconditionally monotone, uniformly smooth basis $\left\{e_{i}\right\}$ and with $\left\{e_{i}^{*}\right\}$ a quasi-uniformly smooth basis for $X^{*}$. Then any nontrivial $M$-ideal in $B(X)$ must contain $K(X)$, and $B(X)$ does not contain any nontrivial $M$-summands.

Proof. Let $f_{i j}$ denote the functional with a one in the $i j$ place and zeros elsewhere. We claim that $\left[f_{i j}:\right.$ all $\left.i, j\right]=K(X)^{*}$. For suppose the contrary, i.e., suppose that there exists an $f \in K(X)^{*}$ which is not a uniform limit of finite matrix elements of $K(X)^{*}$. Since $\left\{e_{i}\right\}$ is shrinking, we can assume w.l.g. that $\left\|f_{n}\right\| \downarrow 1$, where $f_{n}$ is the functional formed from $f$ by deleting the first $n$ rows and columns from the matrix for $f$. Pick $\delta<1$ corresponding to $\varepsilon=1 / 2$ in the definition of a uniformly smooth basis. Then pick $N$ such that $\left\|f_{N}\right\|<(1$ $\left.+\frac{3}{4} \delta\right) /\left(1+\frac{1}{2} \delta\right)$ and choose $T$ and $U$ norm one, disjoint operators (i.e. $\exists m$ such that $t_{i j}=0$ if $i$ or $j>m$ and $u_{i j}=0$ if $i$ or $j<m$ ) with both $f_{N}(T)$ and $f_{N}(U)>1-\delta / 8$. Then,

$$
\frac{f_{N}(T+\delta U)}{\|T+\delta U\|}>\frac{1+\frac{3}{4} \delta}{1+\frac{1}{2} \delta},
$$

which is a contradiction. Hence, $\left[f_{i j}\right.$ : all $\left.i, j\right]=K(X)^{*}$.

Each $f_{i j}$ must be extreme in the unit ball of $K(X)^{*}$, for suppose that $f_{i j}+g$ has a one in the $i j$ place and an $\varepsilon>0$ in the $k l$ place. For this $\varepsilon$, let $\delta$ be the smaller of the smoothness $\delta$ 's for $\left\{e_{i}\right\}$ and $\left\{e_{i}^{*}\right\}$. Then for $T$, the operator with $t_{i j}=1, t_{k l}=\delta$, and zeros elsewhere, we have $\left(f_{i j}+g\right) T=1+\delta \varepsilon$ and $\|T\|<$ $1+\delta \varepsilon$.

In [4] it was shown that if $X$ has an unconditionally monotone, uniformly smooth basis, then $K(X)$ is an HB-subspace of $B(X)$.

Now suppose that $J$ is a nontrivial $M$-ideal in $B(X)$. Each $f_{k l}$ is extreme in the unit ball of $K(X)^{*}$ and hence, by Lemma 1.7 , each $f_{k l}$ must be in $J_{*}$ or 
$J^{\perp}$. Let $T \neq 0$ be in $J$ and pick $f_{i j}: f_{i j}(T) \neq 0$. Then $f_{i j}$ must be in $J_{*}$. Next suppose that some $f_{m n} \in J^{\perp}$. This would contradict the fact that $J$ is an $M$-ideal, since $\left\|f_{i j}+f_{m j}\right\|$ and $\left\|f_{m n}+f_{m j}\right\|$ both have norm less than 2 by Lemma 2.3 and the smoothness hypotheses. Thus, $\left[f_{i j}\right.$ : all $\left.i, j\right] \subset J_{*}$ and by Lemma $1.5 K(X) \subset J$.

$B(X)$ has no nontrivial $M$-summands since, for each norm one $U \in B(X)$, $\exists$ an operator $E_{i j}$ with a one in the $i j$ place and zeros elsewhere such that $\left\|U+E_{i j}\right\|>1$.

COROllary 2.6. For $X=d(a, p)$, any Lorentz sequence space with $1<p<$ $\infty$, the hypotheses of Theorem 2.5 are satisfied.

Proof. To see that the basis $\left\{e_{i}^{*}\right\}$ is quasi-uniformly smooth, note that for each $\delta>0, e_{i}^{*}+\delta e_{j}^{*}$ will achieve its norm on an element of the form $\left(e_{i}+\lambda_{\delta} e_{j}\right) /\left\|e_{i}+\lambda_{\delta} e_{j}\right\|$, such that $\lambda_{\delta} \rightarrow 0$ as $\delta \rightarrow 0$. The basis $\left\{e_{i}\right\}$ is uniformly smooth, since $\|x+y\|^{p} \leqslant\|x\|^{p}+\|y\|^{p}$, whenever $x$ and $y$ are disjoint.

COROLlaRY 2.7. For each $j$ let $X_{j}$ be a space with an unconditionally monotone, uniformly smooth basis $\left\{e_{i}^{j}\right\}$ and a quasi-uniformly smooth basis $\left\{e_{i}^{j *}\right\}$ such that for each $\varepsilon>0$, there is a common smoothness $\delta$ for all $j$. Then the hypotheses of Theorem 2.5 are satisfied for $\left(\sum_{j=1}^{\infty} \bigoplus X_{j}\right)_{l_{p}}$.

\section{BIBLIOGRAPHY}

1. E. M. Alfsen and E. Effros, Structure in real Banach spaces, Ann. of Math. (2) 96 (1972), 98-173.

2. J. Hennefeld, The Arens products and an imbedding theorem, Pacific J. Math. 29 (1969), $551-563$.

3.,$A$ decomposition for $B(X)^{*}$ and unique Hahn-Banach extensions, Pacific J. Math. 46 (1973), 197-199.

4.,$K(X)$ as a subspace of $B(X)$, Indiana Univ. Math. J. (to appear).

5. R. Holmes, B. Scranton, and J. D. Ward, Approximation from the space of compact operators and other M-ideals, Duke Math. J. 42 (1975), 259-269.

6. I. Maddaus, On completely continuous linear transformations, Bull. Amer. Math. Soc. 44 (1938), 279-282.

7. R. R. Smith and J. D. Ward, M-ideal structure in Banach algebras, J. Functional Analysis 27 (1978), 337-349.

8. , M-ideals in $B\left(l_{p}\right)$, Pacific J. Math. (to appear).

Department of Mathematics, Brookiyn College, CUNY, Brooklyn, New York 11210 